\title{
A Confirmed Model to Polymer Core-Shell Structured Nanofibers Deposited via Coaxial Electrospinning
}

\author{
K. Boubaker \\ Unité de Physique des Dispositifs à Semi-Conducteurs, Faculté des Sciences de Tunis, Université de Tunis El Manar, 2092 Tunis, Tunisia \\ Correspondence should be addressed to K. Boubaker, mmbb11112000@yahoo.fr
}

Received 20 August 2012; Accepted 12 September 2012

Academic Editors: B. Hazer and A. V. Popov

Copyright $\odot 2012$ K. Boubaker. This is an open access article distributed under the Creative Commons Attribution License, which permits unrestricted use, distribution, and reproduction in any medium, provided the original work is properly cited.

A model to core-shell structured polymer nanofibers deposited via coaxial electrospinning is presented. Investigations are based on a modified Jacobi-Gauss collocation spectral method, proposed along with the Boubaker Polynomials Expansion Scheme (BPES), for providing solution to a nonlinear Lane-Emden-type equation. The spatial approximation has been based on shifted Jacobi polynomials $P_{T, N}^{(\alpha, \beta)}(x)$ with $\alpha, \beta(-1, \infty), T>0$ was $n$ the polynomial degree. The Boubaker Polynomials Expansion Scheme (BPES) main features, concerning the embedded boundary conditions, have been outlined. The modified Jacobi-Gauss points are used as collocation nodes. Numerical examples are included to demonstrate the validity and applicability of the technique, and a comparison is made with existing results. It has been revealed that both methods are easy to implement and yield very accurate results.

\section{Introduction}

Polymer nanofibers have gained much attention due to their great potential applications, such as filtration, catalysis, scaffolds for tissue engineering, protective clothing, sensors, electrodes electronics applications, reinforcement, and biomedical use [1-6]. Particularly, polymeric nanofibers with core-shell structure have been attractive in the past decades $[4,5]$. Coaxial electrospinning, which has emerged as a method of choice due to the simplicity of the technology and its cost effectiveness, provides an effective and versatile way to fabricate such nanofibers [6-8]. This technique uses a high electric field to extract a liquid jet of polymer solution from the bot core and shell reservoirs. The yielded jet experiences stretching and bending effects due to charge repulsion and, in the process, can reach very small radii. Coaxial electrospinning cannot only be used to spin the unspinnable polymers (polyaramid, nylon, and polyaniline) into ultrafine fibers, but also ensures keeping functionalizing agents like antibacterial and biomolecules agents inside nanofibers [9-11].

In this paper, a mathematical model to coaxial electrospinning dynamics, in a particular setup, is presented.
The model is based on solutions to the related Lane-Emden equation on semi-infinite domains as follows:

$$
u^{\prime \prime}(x)+\frac{a}{x} u^{\prime}(x)=g(x, u(x)) ; \quad 0<x<\infty .
$$

Lane-Emden-type equations model many phenomena in mathematical physics and nanoapplications. They were first published by Lane in 1870 [12], and further explored in detail by Emden [13]. In the last decades, Lane-Emden has been used to model several phenomena such as the theory of stellar structure, quantum mechanics, astrophysics, and the theory of thermionic currents in the neighbourhood of a hot body in thermal equilibrium and the thermal behaviour of an isothermal gaseous sphere [14-18]. Even if LaneEmden problem was numerically challenging because of the singularity behavior at the origin, several methods have been used in order to solve it in semi-infinite domains. Boyd [19] used domain truncation method replacing the semi-infinite domain with $[0, K]$ interval by choosing $K$, sufficiently large, while Shen [20] used spectral-Galerkin approximations based on Laguerre functions to perform analytical solutions and demonstrated that they were stable and convergent with spectral accuracy in the Sobolev spaces. Maday et al. 
[21] proposed a Laguerre type spectral method, Siyyam [22] applied two numerical methods using the Laguerre Tau method, and Guo [23] reformulated the original LaneEmden problem to a singular problem in a bounded domain by variable transformation using the Jacobi polynomials.

This paper is organized as follows. In Section 2 we present an illustrated formulation of the problem, then, in Section 3, we give an overview of the modified Jacobi-Gauss collocation spectral method along with its applications, and in Section 4, we present the fundaments and the application of the Boubaker Polynomials Expansion Scheme (BPES). In Section 5, results are plotted and discussed along wirh comparison with some existing solutions. A conclusion is given in Section 6.

\section{Problem Formulation}

As per Spivak et al. [24, 25], mass balance, linear momentum balance, and electric charge balance equations describe polymer fibers electrospinning process

$$
\begin{gathered}
\nabla \cdot \vec{u}=0, \\
\rho(\vec{u} \cdot \nabla) \vec{u}=\nabla F^{m}+\nabla F^{e}, \\
\nabla \cdot \vec{J}=0,
\end{gathered}
$$

where $\vec{u}$ is the axial velocity, $\vec{J}$ is the electrical current density, $\rho$ is material density, and $F^{m}$ and $F^{e}$ are terms which represent viscous and electric forces, respectively.

Under the assumptions of a steady state jet and a neglectable thermal effort, the electrically generated force is dominant, the monodimensional momentum equation is hence

$$
u \frac{\partial u(x)}{\partial x}=\frac{2 \sigma(x) E(x)}{\rho r},
$$

where $u$ is the modulus of the axial velocity, $r$ is the radius of the jet at axial coordinate $x$ (Figure 1), $\sigma(x)$ is the surface charge density, and $E$ is the exogenous electric field in the axial direction.

By introducing the following charge balance equation:

$$
2 r \sigma(x) u(x)+r^{2} k E(x)=I,
$$

where $I$ is the electrical current intensity and $k$ is a constant which depends only on temperature in the case of an incompressible polymer, it gives

$$
u \frac{\partial u(x)}{\partial x}=\frac{E(x)\left(I-r^{2} k E(x)\right)}{\rho r^{2} u} .
$$

Then, by introducing the variable

$$
y=-6 \operatorname{Ln}(u)
$$

it gives

$$
\frac{\partial y}{\partial x}=-\frac{6 E(x)\left(I-r^{2} k E(x)\right)}{\rho r^{2}} e^{y / 2} .
$$

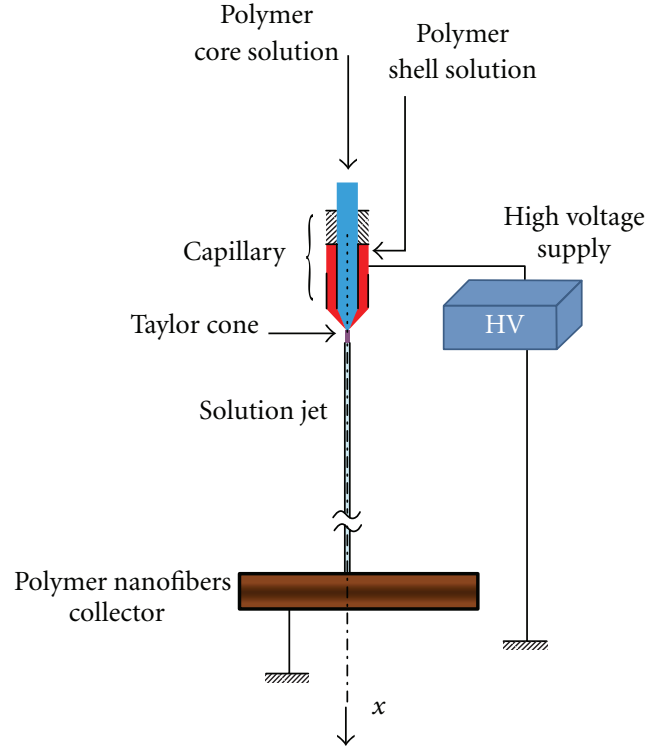

FIgURE 1: Scheme of the studied setup.

By differentiating the last equation, along with assuming weak $r$-dependence of the variable $x$, we have

$$
\begin{gathered}
\frac{\partial^{2} y}{\partial x^{2}}-\left(-\frac{6(d E(x) / d x)}{\rho r^{2}} I e^{y / 2}+\frac{12 d E(x) / d x}{\rho^{2}} E(x) e^{y / 2}\right. \\
\left.-\frac{3 E(x)\left(I-r^{2} k E(x)\right)}{\rho r^{2}} e^{y / 2} \frac{\partial y}{\partial x}\right)=0
\end{gathered}
$$

and by choosing the exogenous electric field profile so that

$$
\begin{gathered}
\frac{3 E(x)\left(I-r^{2} k E(x)\right)}{\rho r^{2}}=\frac{2}{x} e^{-3 \operatorname{Ln}(u) / 2} \\
-\frac{6(d E(x) / d x)}{\rho r^{2}} I e^{y / 2}+\frac{12 d E(x) / d x}{\rho^{2}} E(x) e^{y / 2} \\
=-6 \operatorname{Ln}(u)(-6-4 \operatorname{Ln}(-6 \operatorname{Ln}(u))) e^{-3 \operatorname{Ln}(u) / 2}
\end{gathered}
$$

it gives, by annexing boundary conditions

$$
\begin{aligned}
\frac{\partial^{2} y}{\partial x^{2}}+\frac{2}{x} \frac{\partial y}{\partial x}-6 y(x) & =4 y(x) \operatorname{Ln}(y(x)), \\
y(0) & =1, \\
y^{\prime}(0) & =0 .
\end{aligned}
$$




\section{Modified Jacobi-Gauss Collocation (MJGC) Method}

Let $\alpha>-1, \beta>-1$, and $P_{k}^{(\alpha, \beta)}$ be the standard Jacobi polynomial of degree $k$. We have

$$
\begin{gathered}
P_{k}^{(\alpha, \beta)}(-x)=(-1)^{k} P_{k}^{(\alpha, \beta)}(x), \\
P_{k}^{(\alpha, \beta)}(-1)=\frac{(-1)^{k} \Gamma(k+\beta+1)}{k ! \Gamma(\beta+1)}, \\
P_{k}^{(\alpha, \beta)}(1)=\frac{\Gamma(k+\beta+1)}{k ! \Gamma(\beta+1)} .
\end{gathered}
$$

Then, let $T>0$, then the shifted Jacobi polynomial of degree $k$ on the interval $(0, T)$ is defined by

$$
P_{T, k}^{(\alpha, \beta)}(x)=P_{k}^{(\alpha, \beta)}\left(\frac{2 x}{T}-1\right) .
$$

Consequently, we have

$$
\begin{gathered}
P_{T, k}^{(\alpha, \beta)}(0)=(-1)^{k} \frac{\Gamma(k+\beta+1)}{k ! \Gamma(\beta+1)}, \\
D^{q} P_{T, k}^{(\alpha, \beta)}(0)=\frac{(-1)^{k-q} \Gamma(k+\beta+1)(k+\alpha+\beta+1) q}{T^{q} \Gamma(k-q+1) \Gamma(q+\beta+1)} .
\end{gathered}
$$

For $\alpha=\beta$, one recovers the shifted ultraspherical polynomials (symmetric shifted Jacobi polynomials) and for $\alpha=$ $\beta=\mp 1 / 2, \alpha=\beta=0$ the shifted Chebyshev of the first and second kinds and shifted Legendre polynomials, respectively; for the nonsymmetric shifted Jacobi polynomials, the two important special cases $\alpha=-\beta= \pm 1 / 2$ (shifted Chebyshev polynomials of the third and fourth kinds) are also recovered.

Jacobi-Gauss interpolation starts by denoting $x_{N, j}^{(\alpha, \beta)} 0 \leq$ $j \leq N$, the nodes of the standard Jacobi-Gauss interpolation. The corresponding Christoffel numbers are $\omega_{N, j}^{(\alpha, \beta)} 0 \leq j \leq$ $N$. The nodes of the shifted Jacobi-Gauss interpolation on the interval $(0, T)$ are the zeros of $P_{T, N+1}^{(\alpha, \beta)}(x)$, denoted by $x_{T, N, j}^{(\alpha, \beta)}=(T / 2)^{\alpha+\beta+1} \omega_{N, j}^{(\alpha, \beta)}$. The corresponding Christoffel numbers are $\omega_{T, N, j}^{(\alpha, \beta)}=(T / 2)^{\alpha+\beta+1} \omega_{N, j}^{(\alpha, \beta)}$. Let $S_{N}(0, T)$ be the set of polynomials of degree at most $N$. Thanks to the property of the standard Jacobi-Gauss quadrature, it follows that for any $\Lambda \in S_{2 N+1}(0, T)$

$$
\begin{aligned}
& \int_{0}^{T}(T-x)^{\alpha} x^{\beta} \Lambda(x) d x \\
& =\left(\frac{\tau}{2}\right)^{\alpha+\beta+1} \int_{-1}^{1}(1-x)^{\alpha}(1+x)^{\beta} x^{\beta} \Lambda\left(\left(\frac{T}{2}\right) x_{N, j}^{(\alpha, \beta)}+1\right) d x \\
& =\left(\frac{\tau}{2}\right)^{\alpha+\beta+1} \sum_{j=0}^{N} \omega_{T, N, j}^{(\alpha, \beta)} \Lambda\left(x_{T, N, j}^{(\alpha, \beta)}\right),
\end{aligned}
$$

where $x_{T, N, j}^{(\alpha, \beta)}$ and $\omega_{T, N, j}^{(\alpha, \beta)}$ are the nodes and the corresponding weights of the shifted Jacobi-Gauss-quadrature formula on the interval $(0, T)$, respectively.
For solving (10), we first set

$$
S_{N}(0, T)=\operatorname{span}\left\{P_{T, 0}^{(\alpha, \beta)}(x), P_{T, 1}^{(\alpha, \beta)}(x), \ldots, P_{T, N}^{(\alpha, \beta)}(x)\right\} .
$$

Thus, for any $u \in S_{N}(0, T)$, the norms $\|u\|_{w_{T}^{(\alpha, \beta)}, N}$ and $\|u\|_{w_{T}^{(\alpha, \beta)}}$ coincide.

Associating with this quadrature rule, we denote by $I_{N}^{P_{T}^{(\alpha, \beta)}}$ the shifted Jacobi-Gauss interpolation as follows:

$$
I_{N}^{P_{T}^{(\alpha, \beta)}} u\left(x_{T, N, j}^{(\alpha, \beta)}\right)=u\left(x_{T, N, j}^{(\alpha, \beta)}\right), \quad 0 \leq k \leq N
$$

The shifted Jacobi-Gauss collocation method for solving $(\mathrm{xxx})$ is to seek $z_{N}(x) \in S_{N}(0, T)$, such that

$$
\begin{gathered}
z_{N}^{\prime \prime}\left(x_{T, N, k}^{(\alpha, \beta)}\right)+\left(z^{\prime}\left(x_{T, N, k}^{(\alpha, \beta)}\right)\right)^{2}+\frac{2}{x_{T, N, k}^{(\alpha, \beta)}} z^{\prime}\left(x_{T, N, k}^{(\alpha, \beta)}\right) \\
=4 z\left(x_{T, N, k}^{(\alpha, \beta)}\right)+6 ; \quad k=0,1, \ldots, N-2, \\
z_{N}^{(i)}(0)=0 ; \quad i=0,1 .
\end{gathered}
$$

Let

$$
z_{N}(x)=\sum_{j=0}^{N} a_{j} P_{T, j}^{(\alpha, \beta)}(x), \quad a=\left(a_{0}, a_{1}, \ldots a_{N}\right) .
$$

We first approximate $z(x), z^{\prime}(x)$, and $z^{\prime \prime}(x)$, as (17). By substituting these approximation in (17), we get

$$
\begin{aligned}
& \frac{1}{4} \sum_{j=0}^{N} a_{j}(j+\alpha+\beta+1) P_{T, j-2}^{(\alpha+2, \beta+2)}\left(x_{T, N, k}^{(\alpha, \beta)}\right) \\
& \quad+\frac{1}{2} \sum_{j=0}^{N} a_{j}(j+\alpha+\beta+1) P_{T, j-1}^{(\alpha+1, \beta+1)}\left(x_{T, N, k}^{(\alpha, \beta)}\right)^{2} \\
& \quad+\frac{2}{x_{T, N, k}^{(\alpha, \beta)}} \frac{1}{2} \sum_{j=0}^{N} a_{j}(j+\alpha+\beta+1) P_{T, j-1}^{(\alpha+1, \beta+1)}\left(x_{T, N, k}^{(\alpha, \beta)}\right) \\
& \quad+4 \sum_{j=0}^{N} a_{j} P_{T, j}^{(\alpha, \beta)}\left(x_{T, N, k}^{(\alpha, \beta)}\right)+6 .
\end{aligned}
$$

Next, after using (11) and (13) at $q=1$, we have

$$
\begin{gathered}
\sum_{j=0}^{N}(-1)^{j} \frac{\Gamma(j+\beta+1)}{j ! \Gamma(\beta+1)} a_{j}=0, \\
\sum_{j=0}^{N}(-1)^{j-1} \frac{\Gamma(j+\beta+1)(j+\alpha+\beta+1)}{T(j-1) ! \Gamma(\beta+2)} a_{j}=0 .
\end{gathered}
$$

Finally, from (17), (19), and (20), we get $(N+1)$ nonlinear algebraic equations which can be solved for the unknown coefficients $a_{j}$ by using any standard iteration technique, like Newton's iteration method. Consequently, $z_{N}(x)$ given in (18) can be evaluated, and then the approximate solution of (10) can be obtained. 


\section{The Boubaker Polynomials Expansion Scheme (BPES)}

The Boubaker Polynomials Expansion Scheme (BPES) [2635 ] is a resolution protocol which has been successfully applied to several applied physics and mathematics problems. The BPES protocol ensures the validity of the related boundary conditions regardless main equation features. The BPES is mainly based on Boubaker polynomials first derivatives properties

$$
\begin{gathered}
\left.\sum_{q=1}^{N} B_{4 q}(x)\right|_{x=0}=-2 N \neq 0, \\
\left.\sum_{q=1}^{N} B_{4 q}(x)\right|_{x=r_{q}}=0, \\
\left.\sum_{q=1}^{N} \frac{d B_{4 q}(x)}{d x}\right|_{x=0}=0, \\
\left.\sum_{q=1}^{N} \frac{d B_{4 q}(x)}{d x}\right|_{x=r_{q}}=\sum_{q=1}^{N} H_{q}, \\
\text { with } H_{n}=B_{4 n}^{\prime}\left(r_{n}\right)=\left(\frac{4 r_{n}\left[2-r_{n}^{2}\right] \times \sum_{q=1}^{n} B_{4 q}^{2}\left(r_{n}\right)}{B_{4(n+1)}\left(r_{n}\right)}+4 r_{n}^{3}\right) .
\end{gathered}
$$

Several solution have been proposed through the BPES in many fields such as numerical analysis [26], material characterization [27], theoretical physics [28], mathematical algorithms [29], heat transfer [31, 32], and homodynamics [33-35].

The Boubaker Polynomials Expansion Scheme (BPES) is applied to the system (10) through setting the expression

$$
u(x)=\frac{1}{2 N_{0}} \sum_{k=1}^{N_{0}} \lambda_{k} \times B_{4 k}\left(x r_{k}\right),
$$

where $B_{4 k}$ are the $4 k$-order Boubaker polynomials, $x \in$ $[0,1]$ is the normalized variable, $r_{k}$ are $B_{4 k}$ minimal positive roots, $N_{0}$ is a prefixed integer, and $\left.\lambda_{k}\right|_{k=1 \ldots N_{0}}$ are unknown pondering real coefficients.

Thanks to the properties expressed by (21), boundary condition are trivially verified in advance to resolution process. The system (1) is hence reduced to

$$
\begin{aligned}
& \frac{1}{2 N_{0}} \sum_{k=1}^{N_{0}} \lambda_{k} \times \frac{d^{2} B_{4 k}\left(x r_{k}\right)}{d x^{2}}+\frac{1}{x N_{0}} \sum_{k=1}^{N_{0}} \lambda_{k} \times \frac{d B_{4 k}\left(x r_{k}\right)}{d x} \\
& -\frac{3}{N_{0}} \sum_{k=1}^{N_{0}} \lambda_{k} \times B_{4 k}\left(x r_{k}\right) \\
& \quad=\frac{2}{N_{0}} \sum_{k=1}^{N_{0}} \lambda_{k} \times B_{4 k}\left(x r_{k}\right) \operatorname{Ln}\left(\frac{1}{N_{0}} \sum_{k=1}^{N_{0}} \lambda_{k} \times B_{4 k}\left(x r_{k}\right)\right)
\end{aligned}
$$

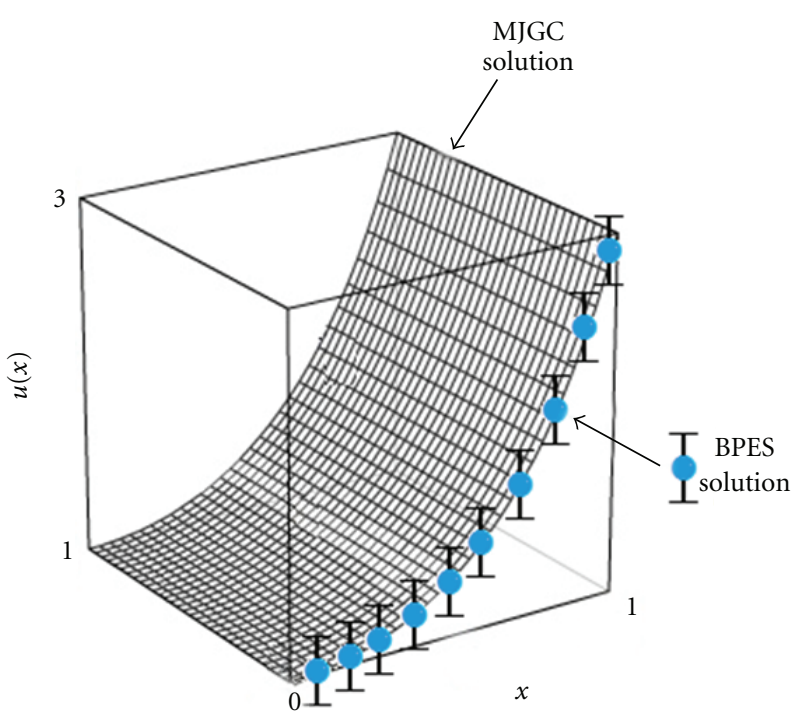

FIGURE 2: Solution plots.

The BPES solution is obtained by determining the nonnull set of coefficients $\left.\tilde{\lambda}_{k}\right|_{k=1 \ldots N_{0}}$ that minimizes the absolute difference $\Delta_{N_{0}}$ as follows:

$$
\Delta_{N_{0}}=\left|\left(\frac{1}{2 N_{0}} \sum_{k=1}^{N_{0}} \tilde{\lambda}_{k} \times \Lambda_{k}\right)-\left(\frac{1}{2 N_{0}} \sum_{k=1}^{N_{0}} \tilde{\lambda}_{k} \times \Lambda_{k}^{\prime}\right)\right|
$$

with

$$
\begin{aligned}
& \Lambda_{k}=r_{k}^{2} \int_{0}^{1} \frac{d^{2} B_{4 k}}{d x^{2}}\left(x \times r_{k}\right) d x \\
& \Lambda_{k}^{\prime}=-r_{k} \int_{0}^{1}\left(\frac{2}{x} \times \frac{d B_{4 k}}{d x}\left(x \times r_{k}\right)\right. \\
&+\left(6+4 \operatorname{Ln}\left(\frac{1}{N_{0}} \sum_{k=1}^{N_{0}} B_{4 k}\left(x \times r_{k}\right)\right)\right) \\
&\left.\times B_{4 k}\left(x \times r_{k}\right)\right) d x .
\end{aligned}
$$

The final solution is hence

$$
u(x)=\frac{1}{2 N_{0}} \sum_{k=1}^{N_{0}} \tilde{\lambda}_{k} \times B_{4 k}\left(x r_{k}\right) .
$$

\section{Results Plots and Discussion}

Plots of the solution obtained by the modified Jacobi-Gauss collocation spectral method are presented in Figure 2, along with BPES solution.

According to the results recorded by Theron et al. [36], He et al. [37, 38], Xu et al. [39], and Thompson et al. [40], velocity at the beginning of the process is jugulated along a short path then is exponentially accelerated. These features are fully verified in the present results (Figure 2). 


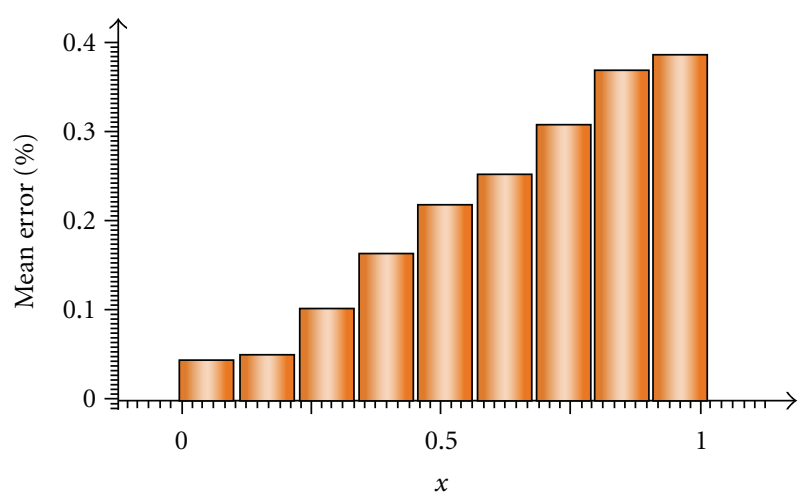

Figure 3: Mean error analyses.

It seems that the two methods ensure the preset boundary condition expressed in (10). From a methodological point of view, the BPES resolution process forces the validity of the boundary conditions prime to establishment of the resolution algorithm while the modified Jacobi-Gauss collocation spectral method takes boundary conditions into account at the same level with the main equation.

Figure 2 displays a velocity profile which matches perfectly the conditions evoked by Zhmayev et al. [41] and Shin et al. [42]. In fact, along an unitary path, jet velocity increases of about 300 percent, which is a favorable condition for fiber formation $[41,42]$.

Additional error analyses yielded a mean relative error below $0.4 \%$ (Figure 3 ).

\section{Conclusion}

The Lane-Emden-type equations describe a variety of phenomena in theoretical physics and astrophysics, including polymer fibres electrospinning process dynamics. In this paper, we tried to give a founded supply to recently proposed related models. Efficient and accurate resolution schemes based on a comparative study of a modified Jacobi-Gauss collocation spectral method and the Boubaker Polynomials Expansion Scheme (BPES) have been used.

Plots and error analysis illustrations were given to demonstrate the validity and applicability of the method. The results show that the model is not very far from real assumptions and considerations.

\section{References}

[1] P. Carlone, G. S. Palazzo, and R. Pasquino, "Modelling of film casting manufacturing process longitudinal and transverse stretching," Mathematical and Computer Modelling, vol. 42, no. 11-12, pp. 1325-1338, 2005.

[2] I. I. Kudish, "Modeling of lubricant performance in Kurt Orbahn tests for viscosity modifiers based on star polymers," Mathematical and Computer Modelling, vol. 46, no. 5-6, pp. 632-656, 2007.

[3] M. Fang, R. P. Gilbert, and X. G. Liu, "A squeeze flow problem with a Navier slip condition," Mathematical and Computer Modelling, vol. 52, no. 1-2, pp. 268-277, 2010.
[4] T. Danno, H. Matsumoto, M. Nasir et al., "Fine structure of PVDF nanofiber fabricated by electrospray deposition," Journal of Polymer Science B, vol. 46, no. 6, pp. 558-563, 2008.

[5] W. Li, C. Liu, Y. Zhou et al., "Enhanced photocatalytic activity in anatase $/ \mathrm{TiO}_{2}$ (B) core-shell nanofiber," Journal of Physical Chemistry C, vol. 112, no. 51, pp. 20539-20545, 2008.

[6] H. Yoshimoto, Y. M. Shin, H. Terai, and J. P. Vacanti, "A biodegradable nanofiber scaffold by electrospinning and its potential for bone tissue engineering," Biomaterials, vol. 24, no. 12, pp. 2077-2082, 2003.

[7] G. E. Wnek, M. E. Carr, D. G. Simpson, and G. L. Bowlin, "Electrospinning of nanofiber fibrinogen structures," Nano Letters, vol. 3, no. 2, pp. 213-216, 2003.

[8] J. A. Matthews, G. E. Wnek, D. G. Simpson, and G. L. Bowlin, "Electrospinning of collagen nanofibers," Biomacromolecules, vol. 3, no. 2, pp. 232-238, 2002.

[9] C. Y. Xu, R. Inai, M. Kotaki, and S. Ramakrishna, "Aligned biodegradable nanofibrous structure: a potential scaffold for blood vessel engineering," Biomaterials, vol. 25, no. 5, pp. 877886, 2004.

[10] M. D. Pierschbacher and E. Ruoslahti, "Cell attachment activity of fibronectin can be duplicated by small synthetic fragments of the molecule," Nature, vol. 309, no. 5963, pp. 3033, 1984.

[11] M. Hakkarainen, "Aliphatic polyesters: abiotic and biotic degradation and degradation products," Advances in Polymer Science, vol. 157, pp. 113-138, 2002.

[12] J. H. Lane, "On the theoretical temperature of the sun under the hypothesis of a gaseous mass maintaining its volume by its internal heat and depending on the laws of gases known to terrestrial experiment," The American Journal of Science and Arts, vol. 50, pp. 57-74, 1870.

[13] R. Emden, Gaskugeln, Teubner, Leipzig, Germany, 1907.

[14] S. Chandrasekhar, An Introduction to the Study of Stellar Structure, Dover Publications, New York, NY, USA, 1957.

[15] H. T. Davis, Introduction to Nonlinear Differential and Integral Equations, Dover Publications, New York, NY, USA, 1962.

[16] O. W. Richardson, The Emission of Electricity from Hot Bodies, Longmans, Green and Company, London, UK, 2nd edition, 1921.

[17] D. C. Biles, M. P. Robinson, and J. S. Spraker, "A generalization of the Lane-Emden equation," Journal of Mathematical Analysis and Applications, vol. 273, no. 2, pp. 654-666, 2002.

[18] G. Bluman, A. F. Cheviakov, and M. Senthilvelan, "Solution and asymptotic/blow-up behaviour of a class of nonlinear dissipative systems," Journal of Mathematical Analysis and Applications, vol. 339, no. 2, pp. 1199-1209, 2008.

[19] J. P. Boyd, Chebyshev and Fourier Spectral Methods, Dover, New York, NY, USA, 2nd edition, 2000.

[20] J. Shen, "Stable and efficient spectral methods in unbounded domains using Laguerre functions," SIAM Journal on Numerical Analysis, vol. 38, no. 4, pp. 1113-1133, 2000.

[21] Y. Maday, B. Pernaud-Thomas, and H. Vandeven, "Reappraisal of Laguerre type spectral methods," Office National D'Etudes et de Recherches Aerospatiales, vol. 6, pp. 13-35, 1985.

[22] H. I. Siyyam, "Laguerre Tau methods for solving higher-order ordinary differential equations," Journal of Computational Analysis and Applications, vol. 3, no. 2, pp. 173-182, 2001.

[23] B. Y. Guo, "Jacobi spectral approximations to differential equations on the half line," Journal of Computational Mathematics, vol. 18, no. 1, pp. 95-112, 2000.

[24] A. F. Spivak, Y. A. Dzenis, and D. H. Reneker, "Model of steady state jet in the electrospinning process," Mechanics Research Communications, vol. 27, no. 1, pp. 37-42, 2000. 
[25] A. F. Spivak and Y. A. Dzenis, "Asymptotic decay of radius of a weakly conductive viscous jet in an external electric field," Applied Physics Letters, vol. 73, no. 21, pp. 3067-3069, 1998.

[26] P. Barry and A. Hennessy, "Meixner-type results for Riordan arrays and associated integer sequences," Journal of Integer Sequences, vol. 13, no. 9, pp. 1-34, 2010.

[27] M. Agida and A. S. Kumar, "A Boubaker polynomials expansion scheme solution to random Love's equation in the case of a rational Kernel," Electronic Journal of Theoretical Physics, vol. 7, no. 24, pp. 319-326, 2010.

[28] A. Yildirim, S. T. Mohyud-Din, and D. H. Zhang, "Analytical solutions to the pulsed Klein-Gordon equation using Modified Variational Iteration Method (MVIM) and Boubaker Polynomials Expansion Scheme (BPES)," Computers and Mathematics with Applications, vol. 59, no. 8, pp. 2473-2477, 2010.

[29] S. Slama, J. Bessrour, M. Bouhafs, and K. B. B. Mahmoud, "Numerical distribution of temperature as a guide to investigation of melting point maximal front spatial evolution during resistance spot welding using boubaker polynomials," Numerical Heat Transfer A, vol. 55, no. 4, pp. 401-408, 2009.

[30] S. Slama, M. Bouhafs, and K. B. Ben Mahmoud, "A boubaker polynomials solution to heat equation for monitoring A3 point evolution during resistance spot welding," International Journal of Heat and Technology, vol. 26, no. 2, pp. 141-146, 2008.

[31] S. A. H. A. E. Tabatabaei, T. Zhao, O. B. Awojoyogbe, and F. O. Moses, "Cut-off cooling velocity profiling inside a keyhole model using the Boubaker polynomials expansion scheme," Heat and Mass Transfer, vol. 45, no. 10, pp. 1247-1251, 2009.

[32] S. Fridjine and M. Amlouk, "A new parameter: an abacus for optimizing PVT hybrid solar device functional materials using the boubaker polynomials expansion scheme," Modern Physics Letters B, vol. 23, no. 17, pp. 2179-2191, 2009.

[33] A. Belhadj, J. Bessrour, M. Bouhafs, and L. Barrallier, "Experimental and theoretical cooling velocity profile inside laser welded metals using keyhole approximation and Boubaker polynomials expansion," Journal of Thermal Analysis and Calorimetry, vol. 97, no. 3, pp. 911-915, 2009.

[34] A. S. Kumar, "An analytical solution to applied mathematicsrelated Love's equation using the Boubaker polynomials expansion scheme," Journal of the Franklin Institute, vol. 347, no. 9, pp. 1755-1761, 2010.

[35] A. Milgram, "The stability of the Boubaker polynomials expansion scheme (BPES)-based solution to Lotka-Volterra problem," Journal of Theoretical Biology, vol. 271, no. 1, pp. 157-158, 2011.

[36] S. A. Theron, E. Zussman, and A. L. Yarin, "Experimental investigation of the governing parameters in the electrospinning of polymer solutions," Polymer, vol. 45, no. 6, pp. 2017-2030, 2004.

[37] J.-H. He, Y. Wu, and W.-W. Zuo, "Critical length of straight jet in electrospinning," Polymer, vol. 46, no. 26, pp. 12637-12640, 2005.

[38] J.-H. He and H.-M. Liu, "Variational approach to nonlinear problems and a review on mathematical model of electrospinning," Nonlinear Analysis: Theory, Methods and Applications, vol. 63, no. 5-7, pp. e919-e929, 2005.

[39] L. Xu, Y. Wu, and Y. Nawaz, "Numerical study of magnetic electrospinning processes," Computers and Mathematics with Applications, vol. 61, no. 8, pp. 2116-2119, 2011.

[40] C. J. Thompson, G. G. Chase, A. L. Yarin, and D. H. Reneker, "Effects of parameters on nanofiber diameter determined from electrospinning model," Polymer, vol. 48, no. 23, pp. 6913-6922, 2007.

[41] E. Zhmayev, D. Cho, and Y. L. Joo, "Nanofibers from gasassisted polymer melt electrospinning," Polymer, vol. 51, no. 18, pp. 4140-4144, 2010.

[42] Y. M. Shin, M. M. Hohman, M. P. Brenner, and G. C. Rutledge, "Experimental characterization of electrospinning: the electrically forced jet and instabilities," Polymer, vol. 42, no. 25, pp. 9955-9967, 2001. 

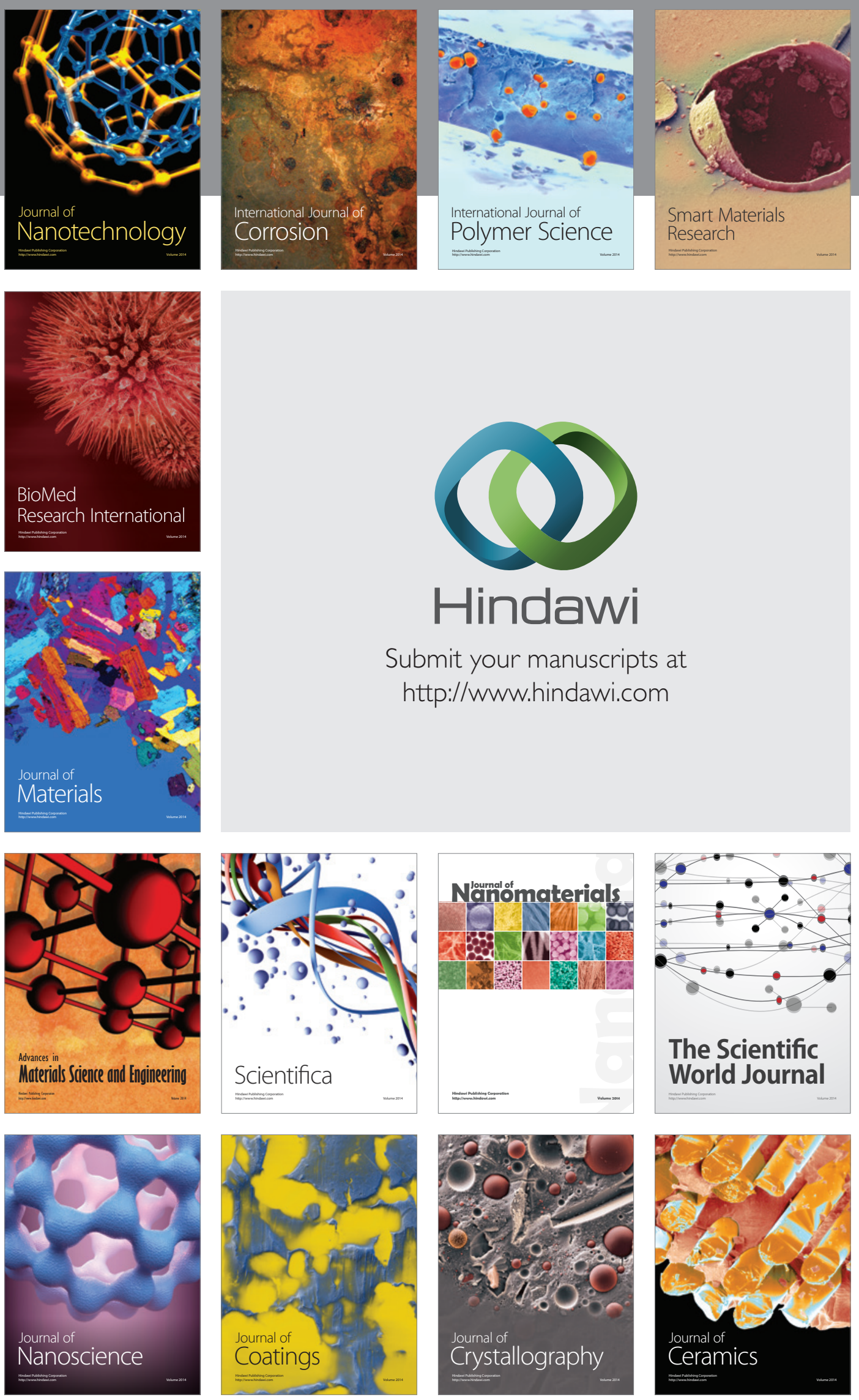

The Scientific World Journal

Submit your manuscripts at

http://www.hindawi.com

\section{World Journal}

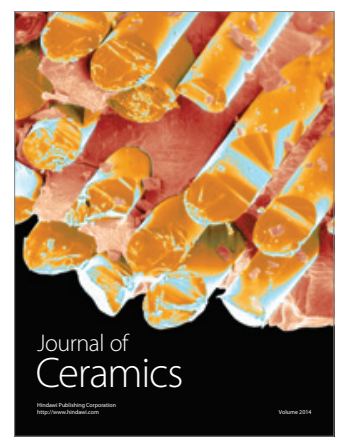

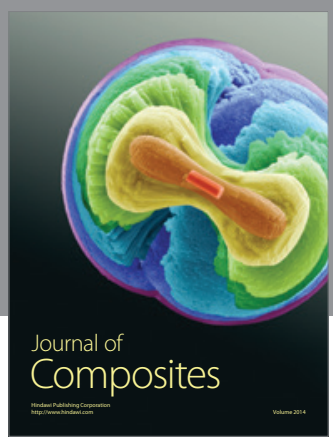
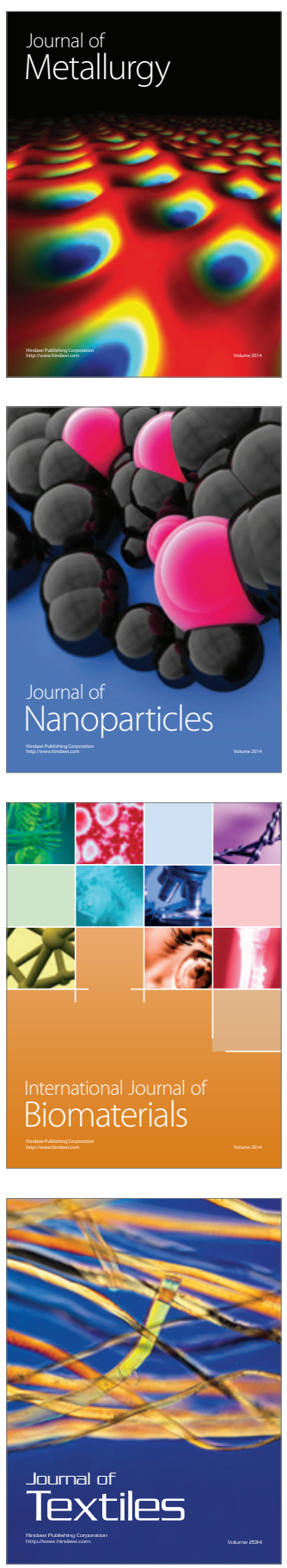